\title{
A case of Systemic Lupus Erythematosis in a male presenting as chronic headache
}

\author{
Edirirweera HMA, Wanniarachchi CG, Sabapathippillai S, Mohideen MR, Herath HMM \\ University Medical Unit, Teaching Hospital Karapitiya, Galle, Sri Lanka
}

Correspondence: Dr. Anushka Ediriweera

e-mail: anushkaediriweera@gmail.com

\section{Introduction}

Systemic Lupus Erythematosus (SLE) is a chronic inflammatory disease that follows a relapsing and remitting course. More than $90 \%$ of cases of SLE occur in women and signs and symptoms mostly are of dermatological, musculoskeletal and haematology related. The prevalence of headache in SLE is about $25 \%$ (1). We report a middle age male presented with chronic headache, later detected to have SLE.

\section{Case report}

A 44-year-old male was admitted with chronic headache and cough for four months duration. The headache was generalized and persisted throughout the day and it wasn't associated with fever, photophobia and visual impairment or vomiting. The cough was nonproductive initially but towards the latter part of illness he had mild haemoptysis. There was no history of significant loss of weight or appetite. He also complained of dark pigmentation of face when exposed to sunlight. Later he had daily fever without any obvious focus of infection.

On physical examination he was pale and found to have left posterior cervical lymphadenopathy and subtle evidence of a malar rash. Neurological examination was normal except the soft exudate found in the right fundus. He had no papilloedema and the rest of the physical examination was normal.

His ESR was $105 \mathrm{~mm}$ in the first hour and CRP was $2.4 \mathrm{~g} / \mathrm{L}$. Full blood count revealed anaemia (Haemoglobin $8.8 \mathrm{~g} / \mathrm{dL}$ ) and thrombocytopenia $\left(52 \times 10^{9}\right)$. Blood picture revealed normochromic normocytic anemia with thrombocytopenia. Direct antibody test was negative and Retic count was normal. He had positive ANA with a titer of 1:1280 and positive dsDNA with a titer of $1: 40$. His contrast enhanced cranial CT was normal. Biopsies cervical lymph node showed necrotizing lymphadenitis.

The diagnosis of SLE was made due to the presence of following four criteria, namely

1) photosensitive rash

2) haematological abnormalities: anaemia and thrombocytopenia

3) positive ANA

4) positive dsDNA

and supportive evidence of SLE such as cytoid bodies and necrotizing lymphadenitis. He was started on prednisolone $45 \mathrm{mg}$ daily and his headache improved gradually following steroids and fever subsided.

\section{Discussion}

SLE is an autoimmune disease in which organs and cells undergo damage initially mediated by tissuebinding auto-antibodies and immune complexes (1). The disease is commonly seen in women $(90 \%)$ in child bearing age. The presentation of illness can be constitutional, musculoskeletal, dermatologic, renal, neuropsychiatric, pulmonary, gastrointestinal, cardiac, hematologic manifestations $(2,3)$. The diagnosis of SLE can be made in the presence of four out of eleven American College of Rheumatology (ACR) criteria (4).

Among patients with SLE headache is seen in $25 \%$ of affected (1). It is not specifically related to the disease severity and the presence of severe headache 
is not indicative of cerebral lupus in the absence of psychosis or seizures (5). Migrainous-type headaches are commoner in patients with SLE than normal population (6). The other rare association of headache in patients with SLE is Idiopathic Intracranial Hypertension (IIH) (7).

Headache remains common and significant symptom among SLE patients, but headache as presenting symptom of it rare. We were able to confirm his condition due to the findings in the history and examination. Generally patients with chronic headaches are subjected to a focus history and limited examination. This case highlights the need of a detailed history and examination in all patients despite their complains. Apart from papilodema which is routinely checked in patients with headache, other subtle changes in the retina and blood vessels also need to be examined.

The other differential diagnosis of chronic headache and high ESR includes temporal arteritis, but the younger age, absence of tender temporal arteries and with highly positive ANA and dsDNA were against this diagnosis. His fundus didn't show evidence of papilloedema and cranial CT also showed no evidence of a cranial pathology.

\section{Conclusion}

Headache is not an uncommon symptom in patients with SLE but some patients with SLE may have chronic headache as a presenting symptom. Detailed history and physical examination would indicate the presence of underlying multisystem diseases such as SLE.

\section{References}

1. Bevra Hannahs Hahn. Systemic Lupus Erythematosus. Chapter 319. Harrison's Principles of Internal Medicine, $18^{\text {th }}$ ed, McGraw Hill; New York, 2012: 2724-31.

2. Christie M Bartels, Herbert S Diamond. Systemic Lupus Erythematosus (SLE) Clinical Presentation eMedicine. Medscape.

3. Edworthy SM. Clinical Manifestations of Systemic Lupus Erythematosus. In: Harris ED, et al, eds. Kelley's Textbook of Rheumatology. $7^{\text {th }}$ ed. Philadelphia, Pa: WB Saunders; 2005: 1201-24.

4. American College of Rheumatology. 1997 Update of the 1982 American College of Rheumatology revised criteria for classification of systemic lupus erythematosus. Available at http://tinyurl.com/1997SLEcriteria. Accessed on March 15, 2012.

5. Sfikakis PP, Mitsikostas DD, Manoussakis MN, Foukaneli D, Moutsopoulos HM. Headache in systemic lupus erythematosus: A controlled Study British Journal of Rheumatology 1998; 37: 300-3.

6. Whitelaw DA, Hugo F, Spangenberg JJ, Rickman R. Headaches in patients with systemic lupus erythematosus: a comparative study. Lupus 2004; 13(7): 501-5.

7. Kuyucu S, Argin A, Kuyucu N, Ozen S. Systemic lupus erythematosus presenting with pseudotumor cerebri: a rare association. TurkJ Pediatr 2007 Jan-Mar; 49(1): 98-101. 